Research Article

\title{
Fractional Metric Dimension of Generalized Sunlet Networks
}

\author{
Muhammad Javaid $\mathbb{D}^{1}{ }^{1}$ Hassan Zafar, ${ }^{1}$ and Ebenezer Bonyah $\mathbb{D}^{2}$ \\ ${ }^{1}$ Department of Mathematics, School of Science, University of Management and Technology, Lahore 54770, Pakistan \\ ${ }^{2}$ Department of Mathematics Education, Akenten Appiah-Menka University of Skills Training and Entrepreneurial Development, \\ Kumasi 00233, Ghana \\ Correspondence should be addressed to Ebenezer Bonyah; ebbonya@gmail.com
}

Received 21 October 2021; Accepted 23 November 2021; Published 21 December 2021

Academic Editor: Gohar Ali

Copyright () 2021 Muhammad Javaid et al. This is an open access article distributed under the Creative Commons Attribution License, which permits unrestricted use, distribution, and reproduction in any medium, provided the original work is properly cited.

\begin{abstract}
Let $N=(V(N), E(N))$ be a connected network with vertex $V(N)$ and edge set $E(N) \subseteq(V(N), E(N))$. For any two vertices $a$ and $b$, the distance $d(a, b)$ is the length of the shortest path between them. The local resolving neighbourhood (LRN) set for any edge $e=\mathrm{ab}$ of $N$ is a set of all those vertices whose distance varies from the end vertices $a$ and $b$ of the edge $e$. A real-valued function $\Phi$ from $V(N)$ to $[0,1]$ is called a local resolving function (LRF) if the sum of all the labels of the elements of each LRN set remains greater or equal to 1 . Thus, the local fractional metric dimension (LFMD) of a connected network $N$ is $\operatorname{dim}_{\text {lf }}(N)=\min \{|\Phi|: \Phi$ is minimal LRF of $N\}$. In this study, LFMD of various types of sunlet-related networks such as sunlet network $\left(S_{m}\right)$, middle sunlet network $\left(\mathrm{MS}_{m}\right)$, and total sunlet network $\left(\mathrm{TS}_{m}\right)$ are studied in the form of exact values and sharp bounds under certain conditions. Furthermore, the unboundedness and boundedness of all the obtained results of LFMD of the sunlet networks are also checked.
\end{abstract}

\section{Introduction}

The problem to find the location number for the connected networks was firstly introduced by Slater in 1975 [1]. Later on, Melter and Harary also studied the concept of location number in networking theory, but they used different term called by metric dimension (MD) [2]. It has been investigated that computing MD is an NP-hard problem [3]. The concept of MD being a graph theoretic parameter is a useful tool in the discovery and verification of the networks [4], allocation of different destinations to robots [5], investigation of percolation in a hierarchical lattice [6], and configuration of the chemical compounds in chemistry [7].

Chartrand et al. established the sharp bounds of MD for the unicyclic networks; they proved that MD of a connected network $N$ is 1 iff $N$ is path network. Furthermore, under certain conditions, by using the concept of $\mathrm{MD}$ on the integer programming problem (IPP), they also found the integral solutions [8]. For the study of the various computational results of $\mathrm{MD}$ for the different connected networks such as Toeplitz, Mobius ladder, lexicographic product of networks, gear networks, and barycentric subdivision of Cayley networks, we refer to [9-13]. In addition, for the study of constant MD of some families of regular, cycle, and prism-related networks and unbounded MD of nanotubes and convex polytopes, see [6, 14-18].

Later on, Currie and Ollerman defined the fractional version of MD to study the nonintegral solution of IPP [19]. Saddiqi and Imran obtained optimal solution of cretin IPP by using this new fractional technique in the field of metricbased dimensions [17]. Arumugam and Matthew formally introduced the term fractional metric dimension (FMD) in graph theory, and they found exact values of FMD for certain connected networks. Moreover, they also characterized all the networks with FMD equal to half of their order. Feng et al. developed computational criteria to compute FMD of the vertex transitive networks in its general form [20]. Recently, Khalidi et al. established sharp bounds 
of FMD for the connected networks [21]. To study the latest developed results on FMD for trees, unicyclic, permutation networks, and product networks obtained under the operation of product (hierarchical, comb, corona, and lexicographic), see [22-26].

The new invariant of MD called by local FMD is defined by Aisyah et al. [27]. Liu et al. computed upper bounds of LFMD for the symmetric and planar networks [28]. For all the connected networks, Javaid et al. established upper bound and improved the lower bound of nonbipartite networks from unity. They also characterized bipartite networks with LFMD as unity [29, 30]. Moreover, Moshin et al. studied LFMD of generalized Petersen networks [31-34]. For more study, we refer to in [34-36].

In this note, our main objective is to compute the sharp bounds and exact values of LFMD for the different generalized sunlet networks such as sunlet network $\left(S_{m}\right)$, middle sunlet network $\left(\mathrm{MS}_{m}\right)$, and total sunlet network $\left(\mathrm{TS}_{m}\right)$, where $m$ is some integral value. In addition, the boundedness and unboundedness of all the obtained results are also investigated. The remaining study is organised as follows. Section 2 contains basic notions. Section 3 has main findings involving LRN sets of LFMD. Section 4 contains the conclusion of this paper.

\section{Preliminaries}

For vertex set $V(N)$ and edge set $E(N) \subseteq(V(N) \times V(N))$, the network $(N=V(N), E(N))$ is constructed as a simple and connected network. For $u, v \in V(N)$, the distance between $u$ and $v$ denoted by $d(u, v)$ is length (number of edges) of the shortest path between them. If each pair of vertices of $N$ is expressed by some path, then $N$ is called connected network. For the further study of preliminary concepts of the subject graph theory, we refer [35].

A vertex $v \in V(N)$ resolves a pair $(w, z)$ of vertices in $N$ if $d(w, v) \neq d(z, v)$. Let $A=\left\{v_{1}, v_{2}, v_{3}, \ldots, v_{m}\right\} \subseteq V(N)$ and $u \in V(N)$; then, $m$-tuple representation of $u$ with respect to $A$ is $r(u \mid A)=\left(r\left(u, v_{1}\right), r\left(u, v_{2}\right), r\left(u, v_{3}\right), \ldots, r\left(u, v_{m}\right)\right)$. If the distinct vertices of $N$ have different representations with respect to $A$, then $A$ is called a resolving set of $N$. Thus, MD of $N$ can be defined by

$$
\operatorname{dim}(N)=\min \{|A|\},
$$

where $A$ is the resolving set of $N$.

Let $u v \in V(N)$; then, local resolving neighbourhood (LRN) is defined as

$$
\mathbb{R}^{\prime}(u v)=\{z \in V(N): d(u, z) \neq d(v, z)\} .
$$

A local resolving function (LRF) is a real-valued function $\Phi: V(N) \longrightarrow[0,1]$ such that $\Phi\left(\mathbb{R}^{\prime}(u v)\right) \geq 1$ for each $\mathbb{R}^{\prime}(u v)$ of $N$, where $\Phi(\mathbb{R}(u v))=\sum_{x \in \mathbb{R}^{\prime}(u v)} \Phi(x)$. A LRF $\Phi$ of $N$ is called minimal if there exists some other function $\Phi^{\prime}: V(N) \longrightarrow[0,1]$ such that $\Phi^{\prime} \leq \Phi$ and $\Phi(u) \neq \Phi^{\prime}(u)$ for at least one $u \in V(N)$ that is not LRF of $N$. Thus, local fractional metric dimension (LFMD) is defined as follows:

$$
\operatorname{dim}_{l f}(N)=\min \{|\Phi|\}
$$

where $|\Phi|=\sum_{u \in V(N)} \Phi(u)$

By using the technique used in [36], now, we define sunlet network $\left(S_{m}\right)$, middle sunlet network $\left(\mathrm{MS}_{m}\right)$, and total sunlet network $\left(\mathrm{TS}_{m}\right)$ as follows.

Let $S_{m}$ be a sunlet network with order and size $2 \mathrm{~m}$, respectively, where $m \geq 3$. It consists of the inner cycle of order $m$, having inner vertices $\left\{v_{i}: 1 \leq i \leq m\right\}$, pendent vertices $\left\{u_{i}: 1 \leq i \leq m\right\}$, and edge set of $E\left(S_{m}\right)=\left\{v_{i} v_{i+1}, u_{i} v_{i}: 1 \leq i \leq m\right\}$, see Figure 1. Middle sunlet network is obtained from sunlet network $S_{m}$ of order $4 m$ and size $5 m$ as $V\left(\mathrm{MS}_{m}\right)=\left\{v_{i}, u_{i}, u_{i}^{\prime}, v_{i}^{\prime}: 1 \leq i \leq m\right\} \quad$ and $E\left(\mathrm{MS}_{m}\right)=\left\{u_{i} u_{i}^{\prime}, v_{i} v_{i}^{\prime}, v_{i}^{\prime} u_{i+1}, v_{i}^{\prime} v_{i+1}^{\prime}, u_{i}^{\prime} v_{i}: 1 \leq i \leq m\right\}$; for details, see Figure 2. The sunlet network $\mathrm{TS}_{m}$ is obtained from middle sunlet network $\mathrm{MS}_{m}$ by adding new edges $\left\{u_{i} v_{i}, v_{i} v_{i+1}: 1 \leq i \leq m\right\}$ with order $4 \mathrm{~m}$ and size $6 \mathrm{~m}$, respectively; for further details, see Figure 3. Now, we define some important results which will be frequently used in the main results as follows.

Theorem 1 (see [29]). For a connected network $N$ and LRN set $\mathbb{R}^{\prime}(e)$ of the edge e of $N$ if $\left|\mathbb{R}^{\prime}(e) \cap A\right| \geq \gamma, \forall e \in E(N)$,

$$
1 \leq \operatorname{dim}_{l f}(N) \leq \frac{|A|}{\gamma},
$$

where $A=\cup\left\{\mathbb{R}^{\prime}(e):\left|\mathbb{R}^{\prime}(e)\right|=\gamma\right\}, \quad \gamma=\min \left\{\left|\mathbb{R}^{\prime}(e)\right|: e \in E\right.$ $(\mathbf{N})\}$, and $2 \leq \gamma \leq|V(N)|$.

Theorem 2 (see [30]). For a connected network $\mathbf{N}$ and LRN set $\mathbb{R}^{\prime}(e)$ of the edge e of $\mathbf{N}$, we have

$$
\frac{|V(N)|}{\beta} \leq \operatorname{dim}_{l f}(N),
$$

where $\beta=\max \left\{\left|\mathbb{R}^{\prime}(e)\right|: e \in E(N)\right\}$ and $2 \leq \beta \leq|V(N)|$.

Corollary 1 (see [30]). For a connected network $N, \mathbb{R}^{\prime}(e)$ as $L R N$ of $e \in E(N), \quad \delta=\max \left\{\left|\mathbb{R}^{\prime}(e)\right|: e \in E(N)\right\}, \quad \gamma=\min$ $\left\{\mathbb{R}^{\prime}(e) \mid: e \in E(\mathbf{N})\right\}, \quad$ and $X=\cup\left\{\mathbb{R}^{\prime}(e):\left|\mathbb{R}^{\prime}(e)\right|=\gamma\right\}$. If $\gamma=\delta$ and $X=V(N)$, then

$$
\operatorname{dim}_{l f}(N)=\frac{|V(N)|}{\delta} .
$$

Theorem 3 (see [29]). If $N$ is a connected bipartite network, then $\operatorname{dim}_{l f}(N)=1$.

\section{Main Result}

In this particular section, we computed LRN sets of generalized sunlet networks and LFMD in the form of exact values and sharp bounds.

3.1. LFMD of Sunlet Network. The resolving neighbourhood sets for each pair of adjacent vertices are classified.

Lemma 1. Let $S_{m}$ with $m \geq 3$ and $m \cong 1(\bmod 2)$ be a sunlet network. Then, for $1 \leq i \leq m$, we have 


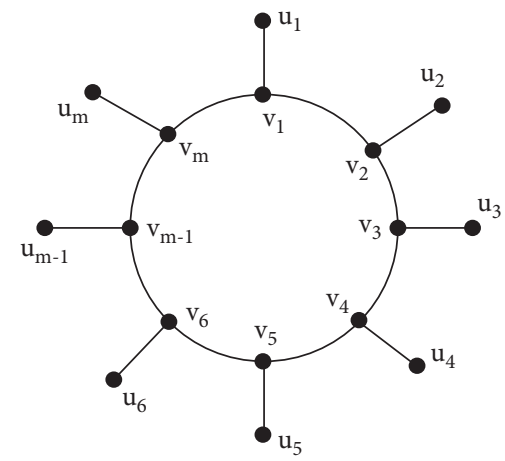

FIgURE 1: Sunlet network $S_{m}$.

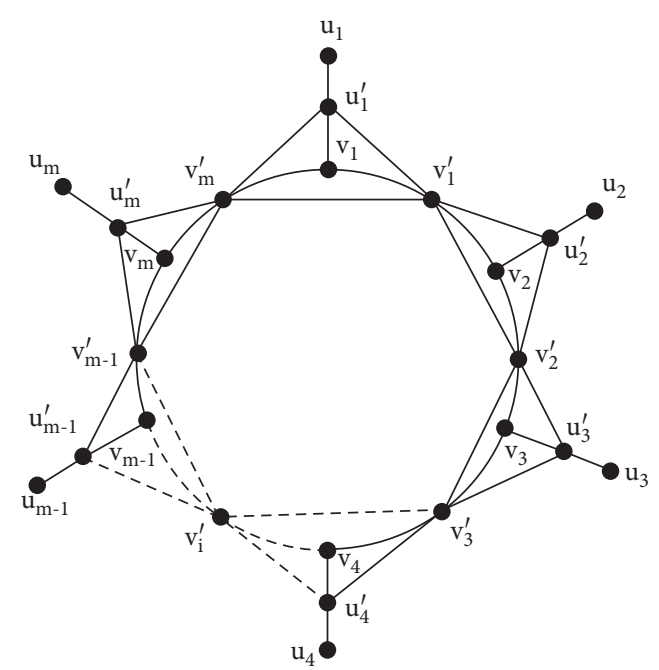

Figure 2: Middle sunlet network $\mathrm{MS}_{m}$.

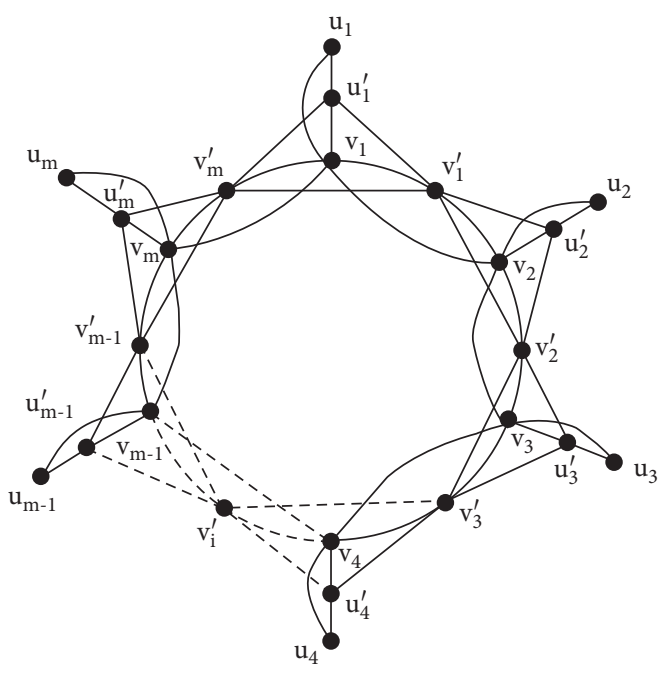

FIgURE 3: Total sunlet network $\mathrm{TS}_{m}$. 
(a) $\left|\mathbb{R}^{\prime}\left(v_{i} v_{i+1}\right)\right|=2 m-2,\left|\mathbb{R}^{\prime}\left(u_{i} v_{i}\right)\right|=2 m, \mid \cup_{i=1}^{m} \mathbb{R}^{\prime}\left(v_{i}\right.$ $\left.v_{i+1}\right) \mid=2 m$, and $\left|\mathbb{R}^{\prime}\left(v_{i} v_{i+1}\right)\right|<\left|\mathbb{R}^{\prime}\left(u_{i} v_{i}\right)\right|$

(b) $\left|\mathbb{R}^{\prime}\left(u_{i} v_{i}\right) \cap \cup_{i=1}^{m} \mathbb{R}^{\prime}\left(v_{i} v_{i+1}\right)\right|=\left|\mathbb{R}^{\prime}\left(v_{i} v_{i+1}\right)\right|$

Proof. Assume that $u_{i}$ pendent and $v_{i}$ are internal vertices, respectively, of $S_{m}$ and $v_{m+1} \cong v_{1}$, where $1 \leq i \leq m$.

(a) Since $\mathbb{R}^{\prime}\left(v_{i} v_{i+1}\right)=V\left(S_{m}\right)-\left\{u_{m+2 i+1 / 2}, v_{m+2 i+1 / 2}\right\}$, this implies that $\left|\mathbb{R}^{\prime}\left(v_{i} v_{i+1}\right)\right|=2 m-2$ and $\mathbb{R}^{\prime}\left(u_{i} v_{i}\right)=$ $V\left(S_{m}\right)$; therefore, $\left|\mathbb{R}^{\prime}\left(v_{i} u_{i}\right)\right|=\left|V\left(S_{m}\right)\right|=2 m$, where $1 \leq i \leq m$. Furthermore, $\left|\cup_{i=1}^{m} \mathbb{R}^{\prime}\left(v_{i} v_{i+1}\right)\right|=2 m$.

(b) Since $\cup_{i=1}^{m} \mathbb{R}^{\prime}\left(v_{i} v_{i+1}\right)=V\left(S_{m}\right)$, therefore, $\mid \mathbb{R}^{\prime}\left(u_{i} v_{i}\right)$ $\cap \cup_{i=1}^{m} \mathbb{R}^{\prime}\left(v_{i} v_{i+1}\right)|=| \mathbb{R}^{\prime}\left(v_{i} v_{i+1}\right) \mid$.

Theorem 4. Let $S_{m}$ be a sunlet network, where $m \geq 3$ and $m \cong 1(\bmod 2)$. Then,

$$
1<\operatorname{dim}_{l f}\left(\mathrm{MS}_{m}\right) \leq m / m-1 .
$$

Proof. To prove the result, we have following cases.

Case 1. For $m=3$, the LRN sets of $S_{m}$ are

$$
\begin{aligned}
& \mathbb{R}_{1}^{\prime}=\mathbb{R}^{\prime}\left(v_{1} v_{2}\right)=V\left(S_{3}\right)-\left\{v_{3}, u_{3}\right\} \\
& \mathbb{R}_{2}^{\prime}=\mathbb{R}^{\prime}\left(v_{2} v_{3}\right)=V\left(S_{3}\right)-\left\{v_{1}, u_{1}\right\} \\
& \mathbb{R}_{3}^{\prime}=\mathbb{R}^{\prime}\left(v_{3} v_{1}\right)=V\left(S_{3}\right)-\left\{v_{2}, u_{2}\right\} \\
& \mathbb{R}_{4}^{\prime}=\mathbb{R}^{\prime}\left(u_{1} v_{1}\right)=V\left(S_{3}\right) \\
& \mathbb{R}_{5}^{\prime}=\mathbb{R}^{\prime}\left(u_{2} v_{2}\right)=V\left(S_{3}\right) \\
& \mathbb{R}_{6}^{\prime}=\mathbb{R}^{\prime}\left(u_{3} v_{3}\right)=V\left(S_{3}\right)
\end{aligned}
$$

From the above LRN sets, $\left|\mathbb{R}_{1}^{\prime}\right|=\left|\mathbb{R}_{2}^{\prime}\right|=\left|\mathbb{R}_{3}^{\prime}\right|=3$ and their cardinalities less than the cardinalities of other LRN sets of $S_{3}$. Hence, the function $h: V\left(S_{3}\right) \longrightarrow[0,1]$ is defined as $h(v)=1 / 4 \forall v \in V\left(S_{3}\right)$ which is an upper LRF; therefore, by Theorem $1, \operatorname{dim}_{l f}\left(S_{3}\right) \leq 3 / 2$.

From the above LRN sets, $\left|\mathbb{R}_{4}^{\prime}\right|=\left|\mathbb{R}_{5}^{\prime}\right|=\left|\mathbb{R}_{6}^{\prime}\right|=6$, and their cardinalities are greater than the cardinalities of other LRN sets of $S_{3}$. Now, the function $h^{\prime}: V\left(S_{3}\right) \longrightarrow[0,1]$ is a lower LRF defined as $h^{\prime}(v)=1 / 6$; hence, by Theorem 2, $\operatorname{dim}_{l f}\left(S_{3}\right) \geq 1$. Since $S_{3}$ is a nonbipartite network, therefore, $\operatorname{dim}_{l f}\left(S_{3}\right)$ must be greater then 1 . Consequently,

$$
1<\operatorname{dim}_{l f}\left(S_{3}\right) \leq 3 / 2 \text {. }
$$

Case 2. In this case, $\left|\mathbb{R}^{\prime}\left(v_{i} v_{i+1}\right)\right|=2 m-2$ and $\mid \cup_{i=1}^{m} \mathbb{R}^{\prime}$ $\left(v_{i} v_{i+1}\right) \mid=2 m$. Furthermore, $\left|\mathbb{R}^{\prime}(y) \cap \cup_{i=1}^{m} \mathbb{R}^{\prime}\left(v_{i} v_{i+1}\right)\right| \geq$ $\left|\mathbb{R}^{\prime}(y)\right| \forall y \in V\left(S_{m}\right)$; hence, the constant function $h: V\left(S_{m}\right) \longrightarrow[0,1]$ is defined as $h(v)=1 / 2 m-$ $2 \forall v \in V\left(S_{m}\right)$ which is an upper LRF; therefore, by Theorem $1, \operatorname{dim}_{l f}\left(S_{m}\right) \leq m / m-1$.

Since $\left|\mathbb{R}^{\prime}\left(u_{i} v_{i}\right)\right|=2 m$ and $\left|\mathbb{R}^{\prime}\left(u_{i} v_{i}\right)\right| \geq\left|\mathbb{R}^{\prime}(y)\right|$ $\forall y \in E\left(S_{m}\right)$, therefore, the function $h^{\prime}: V\left(S_{m}\right) \longrightarrow[0,1]$ is a lower LRF which is defined as $h^{\prime}(v)=1 / 2 m \forall h \in V\left(S_{m}\right)$. Hence, by Theorem $2, \operatorname{dim}_{l f} \geq 1$. Since $S_{m}$ is a nonbipartite network, therefore, $\operatorname{dim}_{l f}\left(\mathrm{MS}_{m}\right)$ must be greater than 1 . Consequently,

$$
1<\operatorname{dim}_{l f}\left(S_{m}\right) \leq \frac{m}{m-1} .
$$

Theorem 5. Let $S_{m}$ be a sunlet network, where $m \geq 4$ and $m \cong 0(\bmod 2)$. Then,

$$
\operatorname{dim}_{l f}\left(S_{m}\right)=1
$$

Proof. Since no cycle in $S_{m}$ is of odd length, therefore, $S_{m}$ is a bipartite network, where $m \cong 0(\bmod 2)$; hence, by Theorem $3, \operatorname{dim}_{l f}\left(S_{m}\right)=1$.

3.2. LFMD of Middle Sunlet Network. The LRN sets for each pair of adjacent vertices are classified.

Lemma 2. Let $M S_{m}$ with $m \geq 3$ be a middle sunlet network. Then, for $1 \leq i \leq m$,

(a) $\left|\mathbb{R}^{\prime}\left(v_{i} u_{i}^{\prime}\right)\right|=3,\left|\mathbb{R}^{\prime}\left(u_{i} u_{i+1}{ }^{\prime}\right)\right|=4 m$, and $\left|\cup_{i=1}^{m} \mathbb{R}^{\prime}\left(v_{i} u_{i}^{\prime}\right)\right|$ $=3 \mathrm{~m}$

(b) $\left|\mathbb{R}^{\prime}\left(v_{i} u_{i}^{\prime}\right)\right| \leq\left|\mathbb{R}^{\prime}(y)\right|$ and $\left|\mathbb{R}^{\prime}(y) \cap \cup_{i=1}^{m} \mathbb{R}^{\prime}\left(v_{i} u_{i}^{\prime}\right)\right| \geq$ $\left|\mathbb{R}^{\prime}\left(v_{i} u_{i}^{\prime}\right)\right| \forall y \in E\left(M S_{m}\right)$

Proof. Assume that $u_{i}$ pendent and $u_{i}^{\prime}, v_{i}^{\prime}$, and $v_{i}$ are internal vertices, respectively, of $\mathrm{MS}_{m}$, where $1 \leq i \leq m$.

(a) Since $\mathbb{R}^{\prime}\left(u_{i}^{\prime} v_{i}\right)=\left\{u_{i}, u_{i}^{\prime}, v_{i}\right\}$, this implies that $\left|\mathbb{R}^{\prime}\left(u_{i}^{\prime} v_{i}\right)\right|=3$ and $\mathbb{R}^{\prime}\left(u_{i} u_{i+1}^{\prime}\right)=V\left(\mathrm{MS}_{m}\right)$; therefore, $\left|\mathbb{R}^{\prime}\left(u_{i} u_{i+1}^{\prime}\right)\right|=\left|V\left(M S_{m}\right)\right|=4 m$, where $\quad 1 \leq i \leq m$. Furthermore, $\left|\cup_{i=1}^{m} \mathbb{R}^{\prime}\left(v_{i} u_{i}^{\prime}\right)\right|=3 m$.

(b) Consider $\mathbb{R}^{\prime}\left(v_{i} v_{i}^{\prime}\right)=\left\{v_{i}, v_{i+1}, v_{i+2}, \ldots, v_{m+2 i-1 / 2}\right.$, $v_{i}^{\prime}, v_{i+1}^{\prime}, v_{i+2}^{\prime}, \ldots, v_{m+2 i-1 / 2}^{\prime}, u_{i+1}, u_{i+2}, \ldots, u_{m+2 i-1 / 2}, u_{i+1}^{\prime}$ , $\left.u_{i+2}^{\prime}, \ldots, u_{m+2 i-1 / 2}^{\prime}\right\}, \mathbb{R}^{\prime}\left(v_{i} v_{i-1}^{\prime}\right)=\left\{v_{i}, v_{m+2 i+1 / 2}, v_{m+2 i}\right.$ $+3 / 2, \ldots, v_{m}, v_{m+2 i+1 / 2}, v_{m+2 i+3 / 2}, v_{m+2 i+5 / 2}, \ldots \ldots \ldots$, $v_{m}^{\prime}, u_{m+2 i+1 / 2}, u_{m+2 i+3 / 2}, u_{m+2 i+5 / 2} \ldots, \quad u_{m}, u_{m+2 i+1 / 2}^{\prime}$, $\left.u_{m+2 i+3 / 2}, u_{m+2 i+5 / 2}, \ldots, u_{m}^{\prime}\right\}, \mathbb{R}^{\prime} \quad\left(u_{i}^{\prime} v_{i}^{\prime}\right)=\left\{v_{i+1}, v_{i+2}\right.$, $v_{i+3}, \ldots, v_{m+2 i-1 / 2}, v_{i}^{\prime}, v_{i+1}^{\prime}, v_{i+2}^{\prime}, \quad v_{i+3}, \ldots, v_{m+2 i-1 / 2}^{\prime}$ $, u_{i}, u_{i+1}, u_{i+2}, u_{i+3}, \ldots, u_{m+2 i-1 / 2}, u_{i}^{\prime}, u_{i+1}^{\prime}, \quad u_{i+2}^{\prime}, u_{i+3}^{\prime}$, $\left.\ldots, u_{m+2 i-1 / 2}^{\prime}\right\}, \mathbb{R}^{\prime}\left(u_{i}^{\prime} v_{i-1}^{\prime}\right)=\left\{v_{m+2 i+1 / 2}, v_{m+2 i+3 / 2}, v_{m+2 i}\right.$ $+5 / 2 \ldots, v_{m}, v_{m+2 i+1 / 2}, v_{m+2 i+3 / 2}, v_{m+2 i+} \quad 5 / 2, \ldots, v_{m}^{\prime}$, $u_{i}, u_{m+2 i+1 / 2}, u_{m+2 i+3 / 2}, \ldots \quad, u_{m}, u_{m+2 i+1 / 2}, u_{m+2 i+3 / 2}$, $\left.\ldots, u_{m}^{\prime}\right\}$, and $\mathbb{R}^{\prime}\left(v_{i}^{\prime} v_{i+1}^{\prime}\right)=V\left(\mathrm{MS}_{m}\right)-\left\{v_{i}, u_{i}^{\prime}, u_{i}\right\}$.

The cardinalities of LRN sets other than $\mathbb{R}^{\prime}\left(u_{i}^{\prime} v_{i}\right)$ are classified in Table 1.

It is clear from Table 1 that $\left|\mathbb{R}^{\prime}\left(u_{i}^{\prime} v_{i}\right)\right| \leq\left|\mathbb{R}^{\prime}(y)\right|$ and $\left|\mathbb{R}^{\prime}(y) \cap \cup_{i=1}^{m} \mathbb{R}^{\prime}\left(v_{i} u_{i}^{\prime}\right)\right| \geq\left|\mathbb{R}^{\prime}\left(v_{i} u_{i}^{\prime}\right)\right| \forall y \in E\left(M S_{m}\right)$.

Theorem 6. Let $M S_{m}$ be a middle sunlet network, where $m \geq 3$.

Then,

$$
1<\operatorname{dim}_{l f}\left(M S_{m}\right) \leq m
$$

Proof. To prove the result, we have the following cases.

Case 3. For $m=3$, the LRN sets of $\mathrm{MS}_{m}$ are 
TABLE 1: Cardinality of each LRN set.

\begin{tabular}{lc}
\hline LRN Set & Cardinality \\
\hline $\mathbb{R}^{\prime}\left(v_{i} v_{i}^{\prime}\right)$ & $2 m>\left|\mathbb{R}^{\prime}\left(u_{i}^{\prime} v_{i}\right)\right|$ \\
$\mathbb{R}^{\prime}\left(v_{i} v_{i-1}^{\prime}\right)$ & $2 m>\left|\mathbb{R}^{\prime}\left(u_{i}^{\prime} v_{i}\right)\right|$ \\
$\mathbb{R}^{\prime}\left(u_{i}^{\prime} v_{i}^{\prime}\right)$ & $2 m+1>\left|\mathbb{R}^{\prime}\left(u_{i}^{\prime} v_{i}\right)\right|$ \\
$\mathbb{R}^{\prime}\left(u_{i}^{\prime} v_{i-1}^{\prime}\right)$ & $2 m>\left|\mathbb{R}^{\prime}\left(u_{i}^{\prime} v_{i}\right)\right|$ \\
\hline
\end{tabular}

$$
\begin{aligned}
& \mathbb{R}_{1}^{\prime}=\mathbb{R}^{\prime}\left(u_{1}^{\prime} v_{1}\right)=\left\{u_{1}^{\prime}, v_{1}, u_{1}\right\} \\
& \mathbb{R}_{2}^{\prime}=\mathbb{R}^{\prime}\left(u_{2}^{\prime} v_{2}\right)=\left\{u_{2}^{\prime}, v_{2}, u_{2}\right\} \\
& \mathbb{R}_{3}^{\prime}=\mathbb{R}^{\prime}\left(u_{3}^{\prime} v_{3}\right)=\left\{u_{3}^{\prime}, v_{3}, u_{3}\right\} \\
& \mathbb{R}_{4}^{\prime}=\mathbb{R}^{\prime}\left(v_{1} v_{1}^{\prime}\right)=\left\{v_{1}, v_{2}, v_{1}^{\prime}, v_{2}^{\prime}, v_{2}, u_{2}^{\prime}\right\} \\
& \mathbb{R}_{5}^{\prime}=\mathbb{R}^{\prime}\left(v_{2} v_{2}^{\prime}\right)=\left\{v_{2}, v_{3}, v_{2}^{\prime}, v_{3}^{\prime}, v_{3}, u_{3}^{\prime}\right\} \\
& \mathbb{R}_{6}^{\prime}=\mathbb{R}^{\prime}\left(v_{3} v_{3}^{\prime}\right)=\left\{v_{3}, v_{1}, v_{3}^{\prime}, v_{1}^{\prime}, v_{1}, u_{1}^{\prime}\right\} \\
& \mathbb{R}_{7}^{\prime}=\mathbb{R}^{\prime}\left(v_{1} v_{3}^{\prime}\right)=\left\{v_{1}, v_{3}, v_{2}^{\prime}, v_{3}^{\prime}, u_{3}, u_{3}^{\prime}\right\} \\
& \mathbb{R}_{8}^{\prime}=\mathbb{R}^{\prime}\left(v_{2} v_{2}^{\prime}\right)=\left\{v_{2}, v_{2}^{\prime}, v_{3}^{\prime}, v_{3}, u_{3}^{\prime}, u_{3}\right\} \\
& \mathbb{R}_{9}^{\prime}=\mathbb{R}^{\prime}\left(v_{3} v_{3}^{\prime}\right)=\left\{v_{3}, v_{3}^{\prime}, v_{1}^{\prime}, v_{1}, u_{1}^{\prime}, u_{1}\right\} \\
& \mathbb{R}_{10}^{\prime}=\mathbb{R}^{\prime}\left(v_{1}^{\prime} v_{3}^{\prime}\right)=\left\{v_{1}^{\prime}, v_{3}^{\prime}, v_{2}, u_{2}^{\prime}, u_{2}, v_{3}, u_{3}^{\prime}, u_{3}\right\} \\
& \mathbb{R}_{11}^{\prime}=\mathbb{R}^{\prime}\left(v_{1}^{\prime} v_{2}^{\prime}\right)=\left\{v_{2}^{\prime}, v_{1}^{\prime}, v_{3}, u_{3}^{\prime}, u_{3}, v_{1}, u_{1}^{\prime}, u_{1}\right\} \\
& \mathbb{R}_{12}^{\prime}=\mathbb{R}^{\prime}\left(v_{2}^{\prime} v_{3}^{\prime}\right)=\left\{v_{2}^{\prime}, v_{1}^{\prime}, v_{3}, u_{3}^{\prime}, u_{3}, v_{1}, u_{1}^{\prime}, u_{1}\right\} \\
& \mathbb{R}_{13}^{\prime}=\mathbb{R}^{\prime}\left(u_{1}^{\prime} u_{1}\right)=V\left(\mathrm{MS}_{3}\right) \\
& \mathbb{R}_{14}^{\prime}=\mathbb{R}^{\prime}\left(u_{2}^{\prime} u_{2}\right)=V\left(\mathrm{MS}_{3}\right) \\
& \mathbb{R}_{15}^{\prime}=\mathbb{R}^{\prime}\left(u_{3}^{\prime} u_{3}\right)=V\left(\mathrm{MS}_{3}\right)
\end{aligned}
$$

From the above LRN sets, $\left|\mathbb{R}_{1}^{\prime}\right|=\left|\mathbb{R}_{2}^{\prime}\right|=\left|\mathbb{R}_{3}^{\prime}\right|=3$ and $\left|\mathbb{R}^{\prime}\left(u_{i}^{\prime} v_{i}\right)\right| \leq\left|\mathbb{R}^{\prime}(y)\right| \forall y \in E\left(\mathrm{MS}_{3}\right) \quad$ and $\left|\mathbb{R}^{\prime}(y) \cap \cup_{i=1}^{3} \mathbb{R}^{\prime}\left(v_{i} u_{i}^{\prime}\right)\right| \geq\left|\mathbb{R}^{\prime}\left(v_{i} u_{i}^{\prime}\right)\right| \forall y \in E\left(\mathrm{MS}_{3}\right)$. Hence, the function $h: V\left(\mathrm{MS}_{3}\right) \longrightarrow[0,1]$ is defined as

$$
h(v)=\left\{\frac{1}{3} i f v \in \bigcup_{i=1}^{3}\left(\mathbb{R}^{\prime}\left(u_{i}^{\prime} v_{i}\right)\right), 0 i f v \in V\left(M S_{3}\right)-\bigcup_{i=1}^{3}\left(\mathbb{R}^{\prime}\left(u_{i}^{\prime} v_{i}\right)\right) .\right.
$$

Therefore, by Theorem $1, \operatorname{dim}_{l f}\left(M S_{3}\right) \leq 3$.

Since $\left|\mathbb{R}_{13}^{\prime}\right|=\left|\mathbb{R}_{14}^{\prime}\right|=\left|\mathbb{R}_{15}^{\prime}\right|=12$ and cardinality of these LRN sets is greater than all other LRN sets, therefore, we define a constant function $h^{\prime}: V\left(M S_{3}\right) \longrightarrow[0,1]$ which is a lower LRF defined by $h^{\prime}(v)=1 / 12$; hence, by Theorem 2, $\operatorname{dim}_{l f} \geq 1$. Also, $\left(\mathrm{MS}_{3}\right)$ is a nonbipartite network; therefore, $\operatorname{dim}_{l f}\left(\mathrm{MS}_{3}\right)$ must be greater then 1. Consequently,

$$
1<\operatorname{dim}_{l f}\left(M S_{3}\right) \leq 3 .
$$

Case 4. In this case, $\left|\mathbb{R}^{\prime}\left(u_{i}^{\prime} v_{i}\right)\right|=3$ and $\left|\cup_{i=1}^{m} \mathbb{R}^{\prime}\left(u_{i} v_{i}\right)\right|=3 m$. Furthermore,

$\left|\mathbb{R}^{\prime}(y) \cap \cup_{i=1}^{m} \mathbb{R}^{\prime}\left(u_{i} v_{i}\right)\right| \geq\left|\mathbb{R}^{\prime}\left(u_{i} v_{i}\right)\right| \forall y \in V\left(\mathrm{MS}_{m}\right) ; \quad$ hence, the constant function $h: V\left(\mathrm{MS}_{3}\right) \longrightarrow[0,1]$ is defined as

$$
h(v)=\left\{\begin{array}{l}
\frac{1}{3} i f v \in \cup_{i=1}^{m}\left(\mathbb{R}^{\prime}\left(u_{i}^{\prime} v_{i}\right)\right), \\
0 i f v \in V\left(M S_{3}\right)-\cup_{i=1}^{m}\left(\mathbb{R}^{\prime}\left(u_{i}^{\prime} v_{i}\right)\right) .
\end{array}\right.
$$

Therefore, by Theorem $1, \operatorname{dim}_{l f}\left(\mathrm{MS}_{m}\right) \leq m$.

Since $\left|\mathbb{R}^{\prime}\left(u_{i}^{\prime} u_{i}\right)\right|=4 m$ and $\left|\mathbb{R}^{\prime}\left(u_{i}^{\prime} u_{i}\right)\right| \geq\left|\mathbb{R}^{\prime}(y)\right| \forall y \in$ $E\left(\mathrm{MS}_{m}\right)$, therefore, a function $h^{\prime}: V\left(\mathrm{MS}_{m}\right) \longrightarrow[0,1]$ is a lower LRF and is defined as $h^{\prime}(v)=1 / 4 m \forall v \in V\left(\mathrm{MS}_{m}\right)$. Hence, by Theorem 2, $\operatorname{dim}_{l f}\left(\mathrm{MS}_{m}\right) \geq 1$. Since $\mathrm{MS}_{m}$ is a nonbipartite network, therefore, $\operatorname{dim}_{l f}\left(\mathrm{MS}_{m}\right)$ must be greater than 1. Consequently,

$$
1<\operatorname{dim}_{l f}\left(\mathrm{MS}_{m}\right) \leq m
$$

3.3. LFMD of Total Sunlet Network. The LRN sets of each pair of adjacent vertices are classified.

Lemma 3. Let $T S_{m}$ with $m \geq 3$ be a total sunlet network. Then, for $1 \leq i \leq m$, we have

(a) $\left|\mathbb{R}^{\prime}\left(v_{i} u_{i}^{\prime}\right)\right|=2, \quad\left|\mathbb{R}^{\prime}\left(u_{i} u_{i+1}{ }^{\prime}\right)\right|=4 m-1$, and $\mid \cup \cup_{i=1}^{m} \mathbb{R}^{\prime}$ $\left(v_{i} u_{i}^{\prime}\right) \mid=2 m$

(b) $\left|\mathbb{R}^{\prime}\left(v_{i} u_{i}^{\prime}\right)\right| \leq\left|\mathbb{R}^{\prime}(y)\right|$ and $\left|\mathbb{R}^{\prime}(y) \cap \cup_{i=1}^{m} \mathbb{R}^{\prime}\left(u_{i}\right)\right| \geq \mid \mathbb{R}^{\prime}$ $\left(u_{i}^{\prime} v_{i}\right) \mid \forall y \in E\left(M S_{m}\right)$

Proof. Assume that $u_{i}$ pendent and $u_{i}^{\prime}$, $v_{i}^{\prime}$, and $v_{i}$ are other vertices, respectively, of $\mathrm{TS}_{m}$, where $1 \leq i \leq m$.

(a) Since $\mathbb{R}^{\prime}\left(u_{i}^{\prime} v_{i}\right)=\left\{u_{i}^{\prime}, v_{i}\right\}$, this implies that $\left|\mathbb{R}^{\prime}\left(u_{i}^{\prime} v_{i}\right)\right|=2$ and $\mathbb{R}^{\prime}\left(u_{i}^{\prime} u_{i}^{\prime}\right)=V\left(\mathrm{TS}_{m}\right)-\left\{v_{i}\right\}$ and $\mathbb{R}^{\prime}$ $\left(u_{i} v_{i}\right)=V\left(\mathrm{TS}_{m}\right)-\left\{u_{i}^{\prime}\right\}$; therefore, $\left|\mathbb{R}^{\prime}\left(u_{i} u_{i}^{\prime}\right)\right|=\left|V\left(\mathrm{TS}_{m}\right)\right|=4 m-1$, where $1 \leq i \leq m$. Furthermore, $\left|\cup_{i=1}^{m} \mathbb{R}^{\prime}\left(v_{i} u_{i}^{\prime}\right)\right|=2 m$.

(b) Consider $\mathbb{R}^{\prime}\left(v_{i} v_{i}^{\prime}\right)=\left\{v_{i}, v_{i+1}, v_{i+2}, \ldots, v_{m+2 i}-1 / 2, v_{i}^{\prime}\right.$, $v_{i+1}{ }^{\prime}, v_{i+2}{ }^{\prime}, \ldots, v_{m+2 i-1 / 2}{ }^{\prime}, u_{i+1}, u_{i+2}, \ldots, u_{m+2 i-1 / 2}, \quad u_{i+1}{ }^{\prime}$, $\left.u_{i+2}^{\prime}, \ldots, u_{m+2 i-1 / 2}^{\prime}\right\}, \quad \mathbb{R}^{\prime}\left(v_{i}^{\prime} v_{i+1}\right)=\left\{v_{i-1}, v_{i}, \quad v_{m+2 i+3 / 2}\right.$, $v_{m+2 i+5 / 2}, \ldots, v_{m}, v_{i}^{\prime}, v_{m+2 i+1 / 2}^{\prime}, \quad v_{m+2 i+3 / 2}, v_{m+2 i+5 / 2}, \ldots$, $v_{m}^{\prime}, u_{i+1}, u_{m+2 i+3 / 2}, \quad u_{m+2 i+5 / 2}, \ldots . ., u_{m}, \quad u_{i}^{\prime}, u_{i+1}^{\prime}$, $\left.u_{m+2 i+3 / 2}, u_{m+2 i+5 / 2}, \ldots, u_{m}^{\prime}\right\}, \quad \mathbb{R}^{\prime}\left(u_{i}^{\prime} v_{i}^{\prime}\right)=\left\{v_{i+1}, v_{i+2}\right.$, $v_{i+3}, \ldots, v_{m+2 i-1 / 2}, \quad v_{i}^{\prime}, v_{i+1}^{\prime}, v_{i+2}^{\prime}, v_{i+3}, \ldots, v_{m+2 i-1 / 2}^{\prime}, u_{i}$, $u_{i+1}, u_{i+2}, u_{i+3}, \ldots, u_{m+2 i-1 / 2}, u_{i}^{\prime}, u_{i+1}{ }^{\prime}, u_{i+2}{ }^{\prime}, \quad u_{i+3}{ }^{\prime}, \ldots$, $\left.u_{m+2 i-1 / 2}^{\prime}\right\}, \mathbb{R}^{\prime}\left(u_{i}^{\prime} v_{i-1}^{\prime}\right)=\left\{v_{m+2 i+1 / 2}, v_{m+2 i+3 / 2}, \quad v_{m+2 i+5 / 2}\right.$ $\ldots, v_{m}, v_{m+2 i+1 / 2}^{\prime}, v_{m+2 i+3 / 2}^{\prime}, v_{m+2 i+5 / 2}, \ldots, v_{m}^{\prime}, \quad u_{i}$, $u_{m+2 i+1 / 2}, u_{m+2 i+3 / 2}, \ldots, u_{m}, \quad u_{m+2 i+1 / 2}, u_{m+2 i+3 / 2}, \ldots$, $\left.u_{m}^{\prime}\right\}$, and $\mathbb{R}^{\prime}\left(v_{i}^{\prime} v_{i+1}^{\prime}\right)=V\left(\mathrm{TS}_{m}\right)-\left\{v_{i}, u_{i}^{\prime}, u_{i}\right\}$.

The cardinalities of the LRN sets other than $\mathbb{R}^{\prime}\left(u_{i}^{\prime} v_{i}\right)$ are classified in Table 2.

It is clear from Table 2 that $\left|\mathbb{R}^{\prime}\left(u_{i}^{\prime} v_{i}\right)\right|$ $\leq\left|\mathbb{R}^{\prime}(y)\right| \forall y \in E\left(\mathrm{TS}_{m}\right)$. Furthermore, $\quad \mid \mathbb{R}^{\prime}(y) \cap \cup_{i=1}^{m}$ $\mathbb{R}^{\prime}\left(u_{i}^{\prime} v_{i}\right)|\geq| \mathbb{R}^{\prime}\left(u_{i}^{\prime} v_{i}\right) \mid$.

Theorem 7. Let $T S_{m}$ be a total sunlet network, where $m \geq 3$. Then,

$$
\operatorname{dim}_{l f}\left(\mathrm{TS}_{m}\right)=m
$$

Proof. To prove the result, we have the following cases.

Case 5. For $m=3$, the $\mathrm{LRN}$ sets of $T S_{m}$ are

$$
\begin{aligned}
& \mathbb{R}_{1}^{\prime}=\mathbb{R}^{\prime}\left(u_{1}^{\prime} v_{1}\right)=\left\{u_{1}^{\prime}, v_{1}\right\} \\
& \mathbb{R}_{2}^{\prime}=\mathbb{R}^{\prime}\left(u_{2}^{\prime} v_{2}\right)=\left\{u_{2}^{\prime}, v_{2}\right\}
\end{aligned}
$$


TABLE 2: Cardinality of each LRN set.

\begin{tabular}{lr}
\hline LRN Set & Cardinality \\
\hline $\mathbb{R}^{\prime}\left(v_{i} v_{i}^{\prime}\right)$ & $2 m>2$ \\
$\mathbb{R}^{\prime}\left(v_{i} v_{i-1}^{\prime}\right)$ & $2 m>2$ \\
$\mathbb{R}^{\prime}\left(u_{i}^{\prime} v_{i}^{\prime}\right)$ & $2 m+1>2$ \\
$\mathbb{R}^{\prime}\left(u_{i}^{\prime} v_{i-1}^{\prime}\right)$ & $2 m>2$ \\
\hline
\end{tabular}

TABLE 3: Bounded and unboundedness of LFMDs.

\begin{tabular}{lcccc}
\hline Network & LFMDs & Lower bound of LFMD when $m \longrightarrow \infty$ & Upper bound of LFMD & Comment \\
\hline$S_{m}$ & $1<\operatorname{dim}_{l f}\left(S_{m}\right) \leq \frac{m}{m-1}$ & 1 & $\lim _{m \longrightarrow 3}\left(\frac{m}{m-1}\right)=1.5$ & Bounded \\
$M S_{m}$ & $1<\operatorname{dim}_{l f}\left(M S_{m}\right) \leq m$ & 1 & $\lim _{m \longrightarrow \infty}(m)=\infty$ & Unbounded \\
\hline
\end{tabular}

$$
\begin{aligned}
& \mathbb{R}_{3}^{\prime}=\mathbb{R}^{\prime}\left(u_{3}^{\prime} v_{3}\right)=\left\{u_{3}^{\prime}, v_{3}\right\} \\
& \mathbb{R}_{4}^{\prime}=\mathbb{R}^{\prime}\left(v_{1} v_{1}^{\prime}\right)=\left\{v_{1}, v_{2}, v_{1}^{\prime}, v_{2}^{\prime}, u_{1}, u_{2}, u_{2}^{\prime}\right\} \\
& \mathbb{R}_{5}^{\prime}=\mathbb{R}^{\prime}\left(v_{2} v_{2}^{\prime}\right)=\left\{v_{2}, v_{3}, v_{2}^{\prime}, v_{3}^{\prime}, u_{2}, u_{3}, u_{3}^{\prime}\right\} \\
& \mathbb{R}_{6}^{\prime}=\mathbb{R}^{\prime}\left(v_{3} v_{3}^{\prime}\right)=\left\{v_{3}, v_{1}, v_{3}^{\prime}, v_{1}^{\prime}, u_{3}, u_{1}, u_{1}^{\prime}\right\} \\
& \mathbb{R}_{7}^{\prime}=\mathbb{R}^{\prime}\left(v_{1} v_{3}^{\prime}\right)=\left\{v_{1}, v_{3}, v_{2}^{\prime}, v_{3}^{\prime}, u_{1}, u_{3}, u_{3}^{\prime}\right\} \\
& \mathbb{R}_{8}^{\prime}=\mathbb{R}^{\prime}\left(v_{2} v_{1}^{\prime}\right)=\left\{v_{2}, v_{1}, v_{1}^{\prime}, v_{3}^{\prime}, u_{2}, u_{1}, u_{1}^{\prime}\right\} \\
& \mathbb{R}_{9}^{\prime}=\mathbb{R}^{\prime}\left(v_{3} v_{2}^{\prime}\right)=\left\{v_{3}, v_{2}, v_{2}^{\prime}, v_{1}^{\prime}, u_{3}, u_{2}, u_{2}^{\prime}\right\} \\
& \mathbb{R}_{10}^{\prime}=\mathbb{R}^{\prime}\left(v_{1}^{\prime} v_{3}^{\prime}\right)=\left\{v_{1}^{\prime}, v_{3}^{\prime}, v_{2}, u_{2}^{\prime}, u_{2}, v_{3}, u_{3}^{\prime}, u_{3}\right\} \\
& \mathbb{R}_{11}^{\prime}=\mathbb{R}^{\prime}\left(v_{1}^{\prime} v_{2}^{\prime}\right)=\left\{v_{2}^{\prime}, v_{1}^{\prime}, v_{3}, u_{3}^{\prime}, u_{3}, v_{1}, u_{1}^{\prime}, u_{1}\right\} \\
& \mathbb{R}_{12}^{\prime}=\mathbb{R}^{\prime}\left(v_{2}^{\prime} v_{3}^{\prime}\right)=\left\{v_{2}^{\prime}, v_{1}^{\prime}, v_{3}, u_{3}^{\prime}, u_{3}, v_{1}, u_{1}^{\prime}, u_{2}\right\} \\
& \mathbb{R}_{13}^{\prime}=\mathbb{R}^{\prime}\left(u_{1}^{\prime} u_{1}\right)=V\left(\mathrm{TS}_{3}\right)-\left\{v_{1}\right\} \\
& \mathbb{R}_{14}^{\prime}=\mathbb{R}^{\prime}\left(u_{2}^{\prime} u_{2}\right)=V\left(\mathrm{TS}_{3}\right)-\left\{v_{2}\right\} \\
& \mathbb{R}_{15}^{\prime}=\mathbb{R}^{\prime}\left(u_{3}^{\prime} u_{3}\right)=V\left(\mathrm{TS}_{3}\right)-\left\{v_{3}\right\} \\
& \mathbb{R}_{16}^{\prime}=\mathbb{R}^{\prime}\left(u_{1} v_{1}\right)=V\left(\mathrm{TS}_{3}\right)-\left\{u_{1}^{\prime}\right\} \\
& \mathbb{R}_{17}^{\prime}=\mathbb{R}^{\prime}\left(u_{2} v_{2}\right)=V\left(\mathrm{TS}_{3}\right)-\left\{u_{2}^{\prime}\right\} \\
& \mathbb{R}_{18}^{\prime}=\mathbb{R}^{\prime}\left(u_{3} v_{3}\right)=V\left(\mathrm{TS}_{3}\right)-\left\{u_{3}^{\prime}\right\}
\end{aligned}
$$

From the above LRN sets $\left|\mathbb{R}_{1}^{\prime}\right|=\left|\mathbb{R}_{2}^{\prime}\right|=\left|\mathbb{R}_{3}^{\prime}\right|=2$ and the cardinalities of these LRN sets which are less than the cardinalities of all other LRN sets, furthermore, $\left|\mathbb{R}(y) \cap \cup_{i=1}^{3} \mathbb{R}\left(u_{i}^{\prime} v_{i}\right)\right| \geq\left|\mathbb{R}\left(u_{i}^{\prime} v_{i}\right)\right| \forall y \in E\left(\mathrm{TS}_{3}\right)$. Hence, the function $h: V\left(\mathrm{TS}_{3}\right) \longrightarrow[0,1]$ is defined as

$$
h(v)=\left\{\frac{1}{2} i f v \in \bigcup_{i=1}^{3}\left(\mathbb{R}^{\prime}\left(u_{i}^{\prime} v_{i}\right)\right), 0 i f v \in V\left(T S_{3}\right)-\bigcup_{i=1}^{3}\left(\mathbb{R}^{\prime}\left(u_{i}^{\prime} v_{i}\right)\right) .\right.
$$

Therefore, $\operatorname{dim}_{l f}\left(\mathrm{TS}_{3}\right)=3$.

Case 6. In this case, $\left|\mathbb{R}^{\prime}\left(u_{i}^{\prime} v_{i}\right)\right|=2$ and $\left|\cup_{i=1}^{m} \mathbb{R}^{\prime}\left(u_{i} v_{i}\right)\right|=2 m$. Furthermore,

$\left|\mathbb{R}^{\prime}(y) \cap \cup_{i=1}^{m} \mathbb{R}^{\prime}\left(u_{i}^{\prime} v_{i}\right)\right| \geq\left|\mathbb{R}^{\prime}\left(u_{i}^{\prime} v_{i}\right)\right| \forall y \in E\left(T S_{m}\right)$. Hence, a constant function $h: V\left(\mathrm{TS}_{m}\right) \longrightarrow[0,1]$ is defined as

$$
h(v)=\left\{\frac{1}{2} i f v \in \bigcup_{i=1}^{m}\left(\mathbb{R}^{\prime}\left(u_{i}^{\prime} v_{i}\right)\right), 0 i f v \in V\left(T S_{m}\right)-\bigcup_{i=1}^{m}\left(\mathbb{R}^{\prime}\left(u_{i}^{\prime} v_{i}\right)\right) .\right.
$$

Therefore,

$$
\operatorname{dim}_{l f}\left(\mathrm{TS}_{m}\right)=m
$$

\section{Conclusion}

In this article, we studied the LFMD of some families generalized sun let networks and formed bounds of LFMDs and computed the exact values of LFMD in some cases as well.

Exact values of LFMD is attained by total sunlet network, $\mathrm{TS}_{m}=m$.

Bounded and unboundedness of LFMDs are illustrated through Table 3.

\section{Data Availability}

The data used to support the findings of this study are included within this article and can be obtained from the corresponding author upon request for more details on the data.

\section{Conflicts of Interest}

The authors declare no conflicts of interest regarding this article.

\section{References}

[1] P. J. Slater, "Leaves of trees," Congressus Numerantium, vol. 14, no. 1, pp. 549-559, 1975.

[2] F. Harary and R. Melter, "On the metric dimension of a graph,” Ars Combinatoria, vol. 2, pp. 19-195, 1976.

[3] M. Garey, R. Johnson, Computers, and Intractability, A Guide to the Theory of NP-Completeness, Freeman, New York, NY, USA, 1979.

[4] Z. Beerliova, F. Eberhard, T. Erlebach et al., "Network discovery and verification," IEEE Journal on Selected Areas in Communications, vol. 24, no. 12, pp. 2168-2181, 2006.

[5] S. Khuller, B. Raghavachari, and A. Rosenfeld, Localization in Graphs, University of Maryland, College Park, MD, USA, 1994.

[6] Y. Shang, "Percolation in a hierarchical lattice," Zeitschrift Natur-Forschung, vol. 67, no. 5, 2012.

[7] M. Johnson, "Structure-activity maps for visualizing the graph variables arising in drug design," Journal of Biopharmaceutical Statistics, vol. 3, no. 2, pp. 203-236, 1993.

[8] G. Chartrand, L. Eroh, M. A. Johnson, and O. R. Oellermann, "Resolvability in graphs and the metric dimension of a graph," Discrete Applied Mathematics, vol. 105, no. 1-3, pp. 99-113, 2000. 
[9] J.-B. Liu, M. F. Nadeem, H. M. A. Siddiqui, and W. Nazir, "Computing metric dimension of Certain families of toeplitz graphs," IEEE Access, vol. 7, pp. 126734-126741, 2019.

[10] M. Ali, G. Ali, M. Imran, A. Q. Baig, and M. K. Shariq, "On the metric dimension of Mobius ladders," Ars Combinatoria, vol. 105, pp. 403-410, 2012.

[11] S. W. Saputro, R. Simanjuntak, S. Uttunggadewa et al., "The metric dimension of the lexicographic product of graphs," Discrete Mathematics, vol. 313, no. 9, pp. 1045-1051, 2013.

[12] S. Imran, M. Siddiqui, M. Imran et al., "Computing the metric dimension of gear graphs," Symmetry, vol. 10, no. 6, p. 209, 2018.

[13] Z. S. Mufti, M. F. Nadeem, A. Ahmad, and Z. Ahmad, "Computation of edge metric dimension of barycentric subdivision of cayley graphs," Italian Journal of Pure and Applied Mathematics, vol. 44, pp. 714-722, 2020.

[14] M. Ali, G. Ali, U. Ali, and M. T. Rahim, "On cycle related graphs with constant metric dimension," Open Journal of Discrete Mathematics, vol. 2, no. 1, pp. 21-23, 2012.

[15] M. Ali, M. T. Rahim, and G. Ali, "On the families of graphs with constant metric dimension," Journal of Prime Research in Mathematics, vol. 8, pp. 95-101, 2012.

[16] M. Imran, A. Q. Baig, and A. Ahmad, "Families of plane graphs with constant metric dimension," Utilitas Mathematica, vol. 88, pp. 43-57, 2012.

[17] H. M. Saddiqi and M. Imran, "Computing metric and partition dimension of 2-dimensional lattices of certain nanotubes," Journal of Computational and Theoretical Nanoscience, vol. 11, no. 12, pp. 2419-2423, 2014.

[18] M. Imran and H. M. A. Siddiqui, "Computing the metric dimension of convex polytopes generated by wheel related graphs," Acta Mathematica Hungarica, vol. 149, no. 1, pp. 10-30, 2016.

[19] J. Currie and O. R. Oellermann, "The metric dimension and metric independence of a graph," Journal of Combinatorial Mathematics and Combinatorial Computing, vol. 39, pp. 157-167, 2001.

[20] M. Feng, B. Lv, and K. Wang, "On the fractional metric dimension of graphs," Discrete Applied Mathematics, vol. 170, pp. 55-63, 2014.

[21] A. H. Alkhaldi, M. K. Aslam, M. Javaid, and A. M. Alanazi, "Bounds of fractional metric dimension and applications with Grid-Related networks," Mathematics, vol. 9, no. 12, p. 1383, 2021.

[22] M. Feng and K. Wang, "On the metric dimension and fractional metric dimension for hierarchical product of graphs," Applicable Analysis and Discrete Mathematics, vol. 7, no. 2, pp. 302-313, 2013.

[23] D. A. Krismanto and S. W. Saputro, "Fractional metric dimension of tree and unicyclic graph," Procedia Computer Science, vol. 74, pp. 47-52, 2015.

[24] Y. Eunjeong, "The fractional metric dimension of permutation graphs," Acta Mathematica Sinica, English Series, vol. 31, pp. 367-382, 2015.

[25] S. W. Saputro, A. Semanicova-Fenovc, M. Baca, and M. Lascsakova, "On fractional metric dimension of comb product graphs," Statistics, Optimization \& Information Computing, vol. 6, no. 1, 2018.

[26] M. Feng and Q. Kong, "On the fractional metric dimension of corona product graphs and lexicographic product graphs," Ars Combinatoria, vol. 138, pp. 249-260, 2018.

[27] S. Aisyah, M. Utoyo, and L. Susilowati, "On the local fractional metric dimension of corona product graphs," IOP
Conference Series: Earth and Environmental Science, vol. 243, pp. 1-4, 2019.

[28] J.-B. Liu, M. K. Aslam, and M. Javaid, "Local fractional metric dimensions of rotationally symmetric and planar networks," IEEE Access, vol. 8, pp. 82404-82420, 2020.

[29] M. Javaid, M. Raza, P. Kumam, and J.-B. Liu, "Sharp bounds of local fractional metric dimensions of connected networks," IEEE Access, vol. 8, pp. 172329-172342, 2020.

[30] M. Javaid, H. Zafar, Q. Zhu, and A. M. Alanazi, "Improved lower bound of LFMD with applications of prism-related networks," Mathematical Problems in Engineering, vol. 2021, Article ID 9950310, 9 pages, 2021.

[31] M. Raza, D. A. Alrowaili, and M. Javaid, "Local fractional metric dimensions of generalized petersen networks," IEEE Access, vol. 9, pp. 74110-74126, 2021.

[32] M. Fehr, S. Gosselin, and O. R. Oellermann, "The metric dimension of Cayley digraphs," Discrete Mathematics, vol. 306, no. 1, pp. 31-41, 2006.

[33] S. Arumugam and V. Mathew, "The fractional metric dimension of graphs," Discrete Mathematics, vol. 312, no. 9, pp. 1584-1590, 2012.

[34] S. Arumugam and V. Mathew, "On fractional metric dimension of graphs," Discrete Mathematics, Algorithms and Applications, vol. 5, no. 4, 2013.

[35] D. B. West, Introduction to Graph Theory, Prentice-Hall, Hoboken, NJ, USA, 2 edition, 2011.

[36] F. Harary, Graph Theory, Narosa Publishing Home, New Delhi, India, 1969. 